\title{
Modalidades vinculares entre padres separados o divorciados e hijos e hijas en la crisis del patriarcado
}

Alicia Bonelli ${ }^{1}$

UADE

\author{
Ensayo \\ Material original autorizado para su primera publicación en el Journal de Ciencias Sociales, Revista Académica de la \\ Facultad de Ciencias Sociales de la Universidad de Palermo.
}

Recibido: 24-11-2016

Aceptado: 14-2-2017

Resumen: Este ensayo versa sobre los cambios en los vínculos entre padres e hijos e hijas a partir de la separación o divorcio, considerando las actuales transiciones y transformaciones tanto en el rol de las mujeres como de los varones en los modelos de crianza.

Dicha reflexión se apoya en los resultados de un sondeo de opinión, realizado en la Fundación Universidad Argentina de la Empresa durante 2016.

Se realizaron 272 encuestas presenciales y 50 entrevistas semidirigidas a hombres divorciados o separados con hijos de entre 0 y 12 años; con estudios terciarios o universitarios; de clase media/alta y residentes en el Área Metropolitana de la Ciudad de Buenos Aires.

Los objetivos de este estudio fueron: comparar el tiempo compartido entre padres e hijos antes y después de producirse la separación y/o divorcio; conocer el grado de compromiso económico, afectivo, educativo y formador respecto a los niños y niñas; reconocer las luchas de poder, que pudieran surgir, entre los excónyuges; identificar si la puesta de límites se ve interferida por la necesidad de apego a los hijos e hijas e investigar cambios en la función paterna.

Como conclusiones se plantea que la función paterna está en transición y por lo tanto en conflicto y contradicción. Es decir, se mantienen algunos rasgos característicos del estereotipo de masculinidad tradicional, por ejemplo, tendencia a definirse como proveedor "fuerte" y evitar tareas asociadas al estereotipo femenino. Sin embargo, estas conductas coexisten en tensión con una mayor participación en las tareas de crianza, con el hecho de

\footnotetext{
${ }^{1}$ Licenciada en Psicología. Docente en la Universidad de Buenos Aires y en la Universidad Argentina de la Empresa. Correo electrónico: aliciabonelli@hotmail.com
} 
compartir la manutención de los hijos e hijas entre ambos padres, con la dificultad para poner límites y, por último, con la expresión del dolor emocional causada por la pérdida de la cotidianeidad con sus hijos e hijas.

Palabras claves: Masculinidad, vínculos paterno-filiales, proceso pos-divorcio, invisibilización.

Abstract: This essay is about the changes in the bonds between parents and children from separation or divorce considering the current transitions and transformations in both the role of women and men in the models of aging.

This reflection is based on the results of an opinion poll conducted at the Universidad Argentina de la Empresa Foundation during 2016. There were 272 face-to-face surveys and 50 semi-directed interviews with divorced and / or separated men with children aged 0 to 12 years; With tertiary or university studies; Of middle/upper class and residing in the Metropolitan Area of the City of Buenos Aires.

The objectives of this study were: to compare times between parents and children before and after separation or divorce; To know the degree of economic, affective, educative and formative commitment with respect to the children; Recognize the power struggles that may arise between former spouses; To identify if the setting of limits is interfered by the need of attachment to the sons and daughters and to investigate changes in the paternal function.

As conclusions, it is argued that the paternal function is in transition and therefore in conflict and contradiction. That is, some characteristics of the stereotype of traditional masculinity are maintained, for example, a tendency to define itself as a "strong" provider and to avoid tasks associated with the female stereotype. However, these behaviors coexist in tension with a greater participation in the tasks of parenting, sharing the support of the sons and daughters between the two parents, difficulty in setting limits and finally with the expression of the emotional pain caused by the loss of daily life with their sons and daughters.

Key words: Masculinity, paternal-filial relationships, post-divorce process, invisibilization.

\section{Introducción}

El siglo XXI, Segunda Modernidad (Giddens, 1998), Sociedad Red o Galaxia Internet (Castells, 2001) o Modernidad líquida (Bauman, 2008), trae consigo procesos de cambio sociocultural y su consecuente transformación, denominada crisis de las instituciones tradicionales. 
Se entiende por 'crisis' una etapa o momento transitorio que se contrapone a lo estacionario o estable de estados anteriores. En una crisis social, el orden de la sociedad se presenta objetivado en productos que no pueden enmarcarse dentro de las categorías conocidas (Berger y Luckmann, 1972), ya que estas se encuentran en revisión, es decir, en proceso de reconstrucción o redefinición.

La familia, en tanto institución tradicional, no queda exenta de dichos cambios, sino que, por el contrario, es un sistema abierto (Watzlawick, 1986) por lo que interactúa con el medio sociocultural, dando lugar así a modificaciones recíprocas.

Como parte de dichos tránsitos, están los cambios en el rol de las mujeres y la crisis del patriarcado (Burin, 2012). Estos son procesos que se articulan en la dinámica de reconstrucción de la estructura de roles de género hegemónica dentro del contexto familiar.

Es dable decir que, en la actualidad, las categorías de lo femenino y lo masculino han entrado en revisión, generando una crisis en el conjunto de relaciones entre varones y mujeres, como también en las relaciones de las mujeres consigo mismas y de los varones consigo mismos, lo cual trae repercusiones en la estructura tradicional familiar.

Una de las transformaciones que se ha registrado en las familias del Área Metropolitana de la Ciudad de Buenos Aires es la disminución del número de matrimonios mientras que ha aumentado el número de divorcios.

Según el informe presentado por la Dirección General de Estadística y Censos (Ministerio de Hacienda GCBA) presentado en 2014, en la Ciudad de Buenos Aires se registra un divorcio por cada dos nuevos matrimonios. Si bien la duración del matrimonio que se disuelve indica que en su mayoría superan los nueve años de convivencia y es bajo el número de matrimonios que se divorcia antes de los cinco años, el porcentaje de parejas que se divorcian durante los primeros doce años de matrimonio es significativo.

Este dato indica que existe una gran cantidad de parejas con hijos e hijas que cursan la primera y segunda infancia (entre 0 y 12 años) y que ya no conviven con los niños y niñas de la misma manera que antes del divorcio. Esta situación implica un cambio en la cotidianeidad de estas familias como también de la manera habitual, propia de la convivencia, de vincularse con ellos.

Este ensayo se propone reflexionar sobre los cambios en la configuración de los vínculos entre padres e hijos e hijas de entre 0 y 12 años a partir de la separación o divorcio, considerando las actuales transiciones y transformaciones tanto en el rol de las mujeres como de los varones en los modelos de crianza. 
Dicha reflexión se apoya en los resultados de un sondeo de opinión, realizado en el Instituto de Investigaciones y Disciplinas Proyectuales de la Fundación Universidad Argentina de la Empresa durante 2016, orientado a conocer cómo se configuran dichos vínculos.

Para tal fin, se realizaron 272 encuestas presenciales y 50 entrevistas semidirigidas a hombres divorciados o separados con hijos de entre 0 y 12 años, con estudios terciarios o universitarios, de clase media/alta y residentes en el Área Metropolitana de la Ciudad de Buenos Aires (AMBA).

El abordaje metodológico cuantitativo se realizó por medio de encuestas con preguntas cerradas con opción de respuesta única y múltiple. La técnica de relevamiento fue por medio de cuestionarios realizados en presencia del encuestador.

El estudio exploratorio se orientó, en primer lugar, a comparar el tiempo compartido, actividades y temas de conversación de los padres con sus hijos e hijas, antes y después de producirse la separación o divorcio; en segundo, a conocer el grado de compromiso económico, afectivo, educativo y formador respecto a los niños; en tercer lugar, a reconocer las luchas de poder, en lo concerniente a la toma de decisiones, que pudieran surgir entre los excónyuges y si estas influyen sobre los comportamientos con los hijos e hijas; en cuarto, a identificar si la puesta de límites se ve interferida por la necesidad de apego a los hijos e hijas y la propia inseguridad; y por último, a investigar cambios en la función paterna.

\section{Argumentación Teórica}

Los ejes que se articulan en esta reflexión y que se presentan en forma sucesiva son los siguientes: en primer lugar, los cambios en los roles de género, femenino y masculino, en el contexto familiar principalmente; en segundo lugar, se plantean algunos de los cambios acaecidos en la esfera del sistema familiar, y, finalmente, se presenta la discusión y reflexión sobre la nueva configuración de los vínculos entre padres separados o divorciados e hijos e hijas a partir de los datos obtenidos del sondeo de opinión previamente citado.

Comenzando por la referencia a los cambios en el rol de las mujeres, se sabe que, a partir de la segunda mitad del siglo XX, la irrupción de las mujeres en espacios laborales, científicos, culturales y políticos, tradicionalmente ocupados por los varones, ha ido redefiniendo y ampliando el lugar tradicional de las mismas como esposa y madre.

A raíz de estas transformaciones, todas las esferas de la vida, tanto pública como privada, comenzaron a modificarse, implicando en estos cambios tanto a varones como a mujeres. 
Como parte de los tránsitos y redefiniciones simultáneas de la subjetividad femenina cabe citar: el tránsito de la heteronomía a la autonomía económica, lo que implica una redefinición y redistribución de las tareas domésticas, los modelos del éxito masculinos y femeninos, la circulación del dinero y las relaciones de poder dentro de la pareja; un tránsito de la heteronomía a la autonomía erótica con la consiguiente redefinición de los lugares de la pasividad y la actividad, de los objetos y sujetos de deseo y, fundamentalmente, de los regímenes de fidelidad en los pactos conyugales y un tránsito de una maternidad como eje central de su proyecto de vida a una maternidad acotada con la consiguiente redefinición de la paternidad y sus incidencias domésticas (Fernández, 2014).

Como correlato de dichos cambios, se observa, particularmente en la esfera del trabajo, que la población económicamente activa, antes con predominio masculino, ha experimentado un proceso denominado "feminización de la fuerza de trabajo" (Ariza y De Olivera, 2001). Esto significa que las mujeres comienzan a ocupar más puestos de trabajo mientras que los varones ven disminuida su participación en el mercado laboral.

Estos movimientos, indudablemente, traen aparejadas redefiniciones de lo que define a lo masculino de manera tradicional. El hombre es "un desconocido" porque cuando se habla de él se lo hace desde una única concepción del mismo, la del hombre patriarcal (Badinter, 1993). Cuando se habla de género, pareciera ser que sólo se habla de la construcción del género femenino y que se "diera por sentado" que el hombre "es asi", o sea, tal como lo define el modelo patriarcal, obviando las condiciones de construcción de su rol que, al igual que el rol de la mujer, es una construcción histórico-social (Waisblat Wainberg y Sáenz Berbejillo, 2011).

La masculinidad tradicional, es decir, la del modelo patriarcal, es definida por el antropólogo David Gilmore según el "modelo de las tres P": preñar, proveer y proteger (citado en Kimmel, 2005).

En consonancia con esto, Fernández (2014) afirma que la construcción de la masculinidad hegemónica reposa sobre una lógica atributiva, binaria y jerárquica. Atributiva, en el sentido de que se le atribuye a lo masculino lo esencialmente humano, es decir, que sólo lo masculino es pensado como positivo y valioso. Lo masculino pasa a constituirse en el modelo de sanidad, honorabilidad, ética y derecho. En cambio, lo femenino se define en términos de negatividad, a partir del modelo masculino positivo. Binaria, en tanto que las diferencias se organizan desde una lógica de oposición; lo femenino se define como "no A" mientras que lo masculino como "A= verdadero". Esto da lugar a la serie de opuestos tales como: activo-pasivo, fuerte-débil, sujeto sexual-objeto sexual, racional-emocional y público-privado. Por último, la lógica jerárquica se refiere a que uno de los términos pasa a ser inferior, complemento o suplemento, negativo de aquello que lo hegemónico señala como uno, como verdadero: la masculinidad. 
La masculinidad se presenta como una relación, además, porque por medio de la masculinidad se nombran determinadas relaciones de poder sobre las que se estructuran posiciones en un sistema de género (Connell, citado en García García, 2009).

Como consecuencia de la naturalización o reificación, en el sentido de Berger y Luckmann (1968), de la masculinidad hegemónica, el varón pasa a concebirse a sí mismo como un ser que debe mostrar y demostrar constantemente su condición de superioridad y verdad. Esto implica sostener una actitud "vigilante" para poder sostener "lo que se espera de él". A su vez, considerando la estructuración jerárquica, donde todo lo que no entra dentro de la masculinidad hegemónica es femenino e inferior, los varones deben estar atentos para no mostrar ningún sentimiento, pasividad, emotividad, ingenuidad, etc., dado que tal situación lo dejaría en una posición inferior femenina (Waisblat Wainberg y Sáenz Berbejillo, 2011).

Otra de las consecuencias, no menos importante, derivada de la construcción de la masculinidad a través del predominio de un código negativo por sobre el positivo, es que la definición de masculinidad se torna inestable, por cuanto cualquier alteración en los modelos de feminidad y en los comportamientos de las mujeres, entraña una crisis de masculinidad, una pérdida de referencia para el código negativo.

En medio de este panorama de cambios, el modelo hegemónico de masculinidad está en crisis, lo que significa que está siendo redefinido. El lugar del varón como protector, proveedor económico de la familia y autoridad es ahora compartido con las mujeres.

Por otro lado, es fundamental, para abordar la temática planteada, considerar los recientes cambios en el Código Civil y Comercial de la Nación (2015). Este reemplaza "la patria potestad", la cual concibe una estructura familiar de tipo verticalista, en la cual los padres tienen poder sobre los hijos e hijas y deciden sobre ellos por "la responsabilidad parental", poniendo el énfasis en la función de cuidado, atención y protección de los niños y niñas. Esto comprende: la manutención, educación, esparcimiento, vestimenta, habitación, asistencia, gastos por enfermedad y los gastos necesarios para adquirir una profesión u oficio.

También se elimina el concepto de "tenencia" y se reemplaza por el de "cuidado personal", que implica una mayor compenetración de ambos padres, sean convivientes o no, para que ambos estén presentes en la vida de sus hijos e hijas. Ante la separación de los padres, se decide que el pequeño o pequeña viva más tiempo en la casa de uno o que pase distintos períodos en cada casa, lo que se denomina "cuidado personal alternado".

Es indudable que dichas modificaciones impactan sobre cómo constituyen estos padres la modalidad vincular con sus hijos e hijas en esta época, lo cual va de la mano con los cambios antes mencionados de los roles masculino y 
femenino, en tanto menos diferenciados en lo que hace al cuidado y responsabilidades con respecto a los hijos, entre otras cosas.

La monogamia secuencial o serial (Acrich, 2016), la cual refiere a que las personas tienden a conformar nuevas parejas monogámicas luego de divorciarse, es otro de los fenómenos de esta época, el cual se encuentra íntimamente ligado a las nuevas configuraciones familiares denominadas "familias ensambladas", en las cuales conviven los hijos e hijas de parejas anteriores de ambos cónyuges. Esta modalidad de familia también tiene repercusiones en la modalidad de vínculo entre padres e hijos e hijas que se constituirá a partir del divorcio. Los niños y niñas, además de elaborar el duelo por la separación de sus padres, podrán encontrarse con la tarea de elaborar la presencia en su cotidianeidad de la nueva pareja de sus padres.

Cabe agregar que, según el nuevo Código Civil y Comercial de la Nación (2015), las nuevas parejas de los padres divorciados que viven con quien tiene a su cargo el cuidado personal del niño, niña o adolescente se denominan "progenitor afín". Estos tienen como obligación cooperar en la crianza, alimentación y educación de los hijos(as) de su pareja.

Otro fenómeno, que viene siendo estudiado en los últimos tiempos, es el denominado "síndrome de alienación parental" (SAP) (Gardner, 1999, citado en Zicavo, 2006), entendido como una alteración que ocurre en algunas rupturas conyugales de alta conflictividad. En ella, los hijos e hijas muestran en sus conductas la censura, crítica y rechazo a uno de sus progenitores, descalificación que es injustificada o exagerada, no presente con anterioridad a la separación de la pareja (Pedrosa y Bouza, 2008), y producto, muchas veces, de cierta violencia psicológica de la mujer hacia el varón.

Con relación, especialmente, al padre, Zicavo (2006) ha detectado, a partir de la clínica, los efectos devastadores que tiene el divorcio sobre este, pues, como vivencia emocional anticipada, suele estar inevitablemente asociado a la pérdida de los hijos, a la ruptura del vínculo relacional, teniendo lugar el inicio de la interrupción de su paternidad (antes construida desde el compromiso) y el menoscabo de espacios generadores de experiencias gratificantes con los hijos e hijas.

También es relevante destacar que en muchas oportunidades los padres "utilizan" a los hijos e hijas como confidentes y depositarios de detalles que corresponden a la intimidad del rol de pareja, como salvadores y fuente de apoyo emocional hacia el padre o la madre o, en otros casos, procuran contribuir con la compresión y readaptación del estilo de vida de los hijos e hijas, luego de la separación o divorcio. 
Visto lo anterior, cabe preguntarse: ¿cómo manejan los padres el malestar de los hijos e hijas? A saber, la culpa, rabia, impotencia, tristeza que conlleva la separación de sus padres. ¿Cómo los padres, pese al divorcio, llegan a acuerdos básicos? Estos son normas mínimas que toda familia requiere para vivir con hábitos y creciendo con límites que luego se trasladan a las instancias donde se desenvuelven: escuela, colegio, academias, universidad, trabajo, el nuevo hogar que formen, etc. Por ejemplo: horarios y actividades entre semana, el fin de semana, manejo de la tecnología, la intervención de los abuelos y abuelas y los límites de la información que se les da, entre otros.

Ahora bien, considerando los resultados obtenidos en el sondeo de opinión citado, se destaca, en cuanto a las características de la muestra, que más de la mitad de los participantes (56\%) se separó o divorció hace menos de 3 años. Un 36\% asegura haber tomado la decisión de separarse o divorciarse. Sólo un $25 \%$ sostuvo que la decisión la tomó su expareja. Más del 64\% tiene un solo hijo/a menor de 12 años y un 31\% tiene 2 hijos/as. El 91\% afirma que sus hijos/as son de una misma expareja.

Con respecto al vínculo que estos padres tienen con sus hijos e hijas, se registró que casi la mitad de la muestra (46\%) afirma que los niños o las niñas duermen en su casa 1 o 2 vez por día en la semana, por lo general durante los fines de semana. Sólo un 10\% tiene a sus hijos e hijas más de 5 días por semana durmiendo en casa.

La mayoría (52\%) considera que hubo cambios en cuanto a las actividades que realizaba con sus hijos e hijas antes de la separación comparadas con las que realiza ahora. De este porcentaje, el $24 \%$ piensa que hubo un cambio "muy fuerte".

En las entrevistas, los participantes expresaron el deseo de compartir más tiempo con sus hijos e hijas del habitual. También manifestaron que esta diferencia entre el tiempo actual compartido y el anterior a la separación era, para ellos, "uno de los efectos de la separación más dolorosos".

Así mismo, la mayoría (65\%) dice tener un espacio destinado a sus hijos e hijas en su casa con la intención de constituir un ámbito que pueda ser sentido como "un hogar" para los niños.

Este anhelo, por parte de estos padres, se manifiesta en la representación que tienen acerca de la percepción de los hijos e hijas de su casa actual.

Es así como un 58\% considera que sus hijos e hijas poseen una imagen de "hogar" acerca de su casa. Sólo un $10 \%$ considera que los niños y niñas no tienen esa imagen. 
En cuanto a la representación que estos padres tienen acerca de la manera como la separación afectó a los hijos/as, la mayoría (66\%) considera que ellos se vieron afectados emocionalmente por la separación o el divorcio; la mitad de esta mayoría habla del tema con sus hijos/as.

Estos datos ponen de manifiesto que los padres participantes comparten menos tiempo con sus hijos/as que aquel compartido antes de la separación o divorcio, lo cual se encuentra relacionado con lo planteado por Zincavo (2006), quien sostiene que el proceso posdivorcio o postseparación acarrea, a nivel real y vivencial, una ruptura impuesta de la figura paterna con los hijos/as. Esto implica que, de forma inevitable, ocurre un nivel de pérdida o alejamiento del padre, con el correspondiente costo afectivo que esta realidad trae consigo. La sociedad le impone, por lo general, al padre la lejanía, lo cual en muchas ocasiones va acompañado de añoranza, gran sentimiento de dolor y sentimiento de culpa por el malestar causado a sus hijos e hijas, pues se trata de separarse precisamente de lo que más ama, de lo que jamás planificó ni previó distanciarse: los hijos/as.

En cuanto a las actividades que comparten con ellos, los resultados revelan que un $66 \%$ dice que juega con sus niños/as; más de la mitad (56\%), que come; menos de la mitad (49\%) dice hablar con ellos/as; y un $45 \%$ dice mirar televisión con ellos/as. Solo el $28 \%$ revisa las tareas escolares con sus hijos e hijas.

Se observa, entonces, que los varones de la muestra tienden a evitar la realización de tareas asociadas con el imaginario tradicional femenino (principalmente tareas escolares e higiene personal); sin embargo, sí dicen prepararles la comida y contenerlos emocionalmente.

Con relación a los temas de conversación que comparten con sus pequeños/as, la mayoría (57\%) asegura que casi nunca o nunca habla con sus hijos/as sobre sus propias preocupaciones, malestares o alegrías. Sólo un $24 \%$ dice hablar sobre ello siempre o casi siempre, mientras que un escaso $35 \%$ prefiere hablar con sus hijos e hijas sobre sus sentimientos.

El modelo de masculinidad hegemónico sigue estando presente, en tanto marco de referencia, en las respuestas de los participantes, ya que no se identifican mayormente con las tareas asignadas a las mujeres y evitan mostrarse "débiles" o "sentimentales", sosteniendo el paradigma del hombre "protector y fuerte".

Lo que se designa como masculinidad hegemónica es una representación de masculinidad donde se destacan mandatos irrevocables para el varón como el no doblegarse al dolor, el no pedir ayuda bajo ninguna circunstancia, el no temerle a nada, el no llorar, ser violento y agresivo. Esto indica que lo masculino se asocia en muchas ocasiones a la expropiación de la posibilidad de expresar libremente sentimientos y emociones, a la privación de disfrutar 
plenamente del cariño de sus hijos e hijas, lo que trae como consecuencia el distanciamiento de espacios vitales como el de la familia (Ruble, citado en Hyde, 1995; Quaresma da Silva, Ulloa y Sperling, 2013; Rivero, 2005).

Otra dimensión indagada en este sondeo y que se encuentra íntimamente relacionada con lo planteado previamente es la referida al aporte económico que estos padres dan a la madre para la manutención de los hijos e hijas. Se encontró que la mayoría (52\%) afirma cubrir todos los gastos de los niños y niñas, mientras que un $29 \%$ dice cubrir solamente alimentos, ropa y útiles escolares.

El $46 \%$ de los participantes afirma que su expareja aporta mucho económicamente para el cuidado de sus hijos e hijas. El 15\% sostiene que no realiza ningún aporte y el 39\% que aporta poco.

Al respecto, Brannon y David (citados en Segara y Carabí, 2000) enuncian, coincidiendo con los datos previamente citados, cuatro factores que caracterizan a la masculinidad hegemónica: carencia de rasgos feminoides, seguridad en sí mismos (no querer mostrarse sensibles, con problemas); ser exitosos y ganar buen dinero (ser el sostén económico de sus hijos).

El mandato de ser el "padre ideal" sigue estando vigente en estos padres, de allí que intentan mostrar una imagen que sea congruente con este ideal: la mayoría encarna el rol de proveedor.

Sin embargo, se registran algunos cambios en cuanto al rol masculino tradicional, tales como la participación más activa en las tareas de crianza (preparación de alimentos, contención emocional, lavado de la ropa, cuidado cuando están enfermos) que otrora eran asignadas a las mujeres exclusivamente.

Estos cambios en los roles también se observan en los resultados que siguen: un $45 \%$ dice que sus hijos e hijas tienen ropa en su casa y que ellos se encargan de lavarla y ordenarla; un 35\% afirma que sus hijos e hijas no tienen su propia ropa en su casa y que la traen de la casa de su madre. En lo referente al cuidado cuando están enfermos: casi la mitad (45\%) de los encuestados sostiene que sus hijos e hijas van igualmente a sus casas cuando tienen un pequeño malestar físico. Sólo un $20 \%$ dice que se quedan al cuidado de sus madres.

El hecho de que se detecte una participación más activa de parte del género masculino con respecto a las tareas de crianza de los hijos e hijas se relaciona con los aportes de Ramos Torres y Tobio Soler (citados en Carrasco y Mina García, 2005), quienes sostienen que, en lo concerniente a los cambios en la distribución de las tareas domésticas, en particular en tareas asociadas al cuidado de los niños y niñas, el hombre se ha vuelto más participativo en la medida que el nivel socioeconómico del trabajo de ambos es más elevado y recupera un imaginario del rol de género más igualitario. Esto se observa en el aporte económico de las mujeres; si bien, al decir de los padres, ellos 
aportan el dinero para cubrir la mayoría de los gastos, las mujeres realizan un aporte muy significativo, casi igual a los varones.

La crisis del patriarcado entraña, entre otros aspectos, cambios en lo referido al lugar de los varones en tanto autoridad en la familia, la puesta de límites ya no es un rol exclusivo de la figura paterna, sino que el mismo es compartido con las madres (Burin, 2012). Esto acarrea una serie de conflictos a partir de opiniones y puntos de vista diferentes en cuanto a temas vinculados a la crianza de los hijos e hijas.

Sólo un 22\% dice no discutir sobre los límites de sus hijos e hijas con su expareja y un $22 \%$ dice que surgen conflictos siempre o casi siempre. La mayoría restante (56\%) afirma que los desacuerdos surgen con una frecuencia que identificaron con el término "a veces".

Al respecto, los resultados obtenidos indican que las discusiones con la madre son sobre diversidad de temas: hábitos cotidianos (30\%); modales, valores o normas (28\%); aporte económico (27\%) y uso de la tecnología (13\%).

Pese a que surgen desacuerdos en este terreno, la amplia mayoría (85\%) dice no hacer nunca o casi nunca comentarios ante los niños y niñas acerca de cuestiones en las que no acuerda con su expareja para evitar exponerlos a una situación dolorosa e incómoda. Por esta razón, buscan armonizar los estilos de crianzas y de manejo de conductas de los hijos/as en los dos nuevos hogares que ahora tendrán (el de la madre y el del padre), evitando cuestionar y descalificar frente los hijos e hijas el proceder del otro progenitor (Guzmán y Constenla, 2012).

En cuanto a cómo se perciben los padres a sí mismos a la hora de poner límites, sólo un 30\% sostuvo que le resulta difícil poner límites a los hijos/as, mientras que el 62\% afirmó poner los límites con naturalidad. Sin embargo, pese a que los padres muestran una imagen de sí consistente con el de encarnar el rol de "autoridad paterna", la mayoría de los padres de la muestra (51\%) afirmó que frente a las demandas de sus hijos/as tratan de satisfacerlos en todo los que les piden y un $49 \%$ sostuvo que sólo los satisfacen en algunas cosas que consideran necesarias.

Se considera, a partir de dichos datos, que estos padres se encuentran ante una situación conflictiva: por un lado, mantener la imagen de un "padre con autoridad" y por el otro ser "compañero y amigo" de sus hijos e hijas.

En las entrevistas, se registró que tratan de darles "todo y más de lo que pueden", para que los niños estén contentos con ellos; puesto que están poco tiempo con los hijos e hijas, quieren disfrutarlos.

En los casos en que la decisión de la separación provino del padre, los varones entrevistados coincidieron en decir que demoraron la misma por los hijos/as. Uno de los participantes, al referirse a la etapa previa a tomar la 
decisión de separarse, comentó: "Me costaba pensar que no lo iba a ver todos los días al llegar a casa" (Juan, 35 años).

Bauman (2008) afirma que, en la sociedad posmoderna, el hijo es el único vínculo que perdura a lo largo de la vida, un reaseguro contra la soledad, mientras que otros vínculos interpersonales son efímeros y "descartables". Por eso es válido considerar que el hijo pasa a convertirse en un reaseguro afectivo también para estos padres. Al decir de Bowlby (1989), una figura de apego.

Por otro lado, la pérdida de los hijos y el menoscabo de espacios generadores de experiencias gratificantes con los niños que ocurre tras la separación (Zicavo, 2006) explica, en parte, esta ambivalencia presentada por estos adultos: por un lado, seguir desempeñando el rol de autoridad y ponerles límites, los cuales son, en muchas ocasiones, generadores de conflicto con la madre, y por otro lado, consentirlos para disfrutar el poco tiempo que comparten y asegurarse así la permanencia del vínculo que más valoran.

Algo que surgió en las entrevistas y que es factible vincular con la hipótesis planteada sobre la necesidad afectiva del adulto que cubre el niño y/o niña, es que muchos entrevistados que no formaron pareja comentan que, a pesar de tener una cama en su hogar para el niño/a, expresaron ideas como la siguiente: "Muchas veces dormimos juntos, porque nos quedamos viendo la tele y además me gusta abrazarlo a la noche cuando duerme" (Pedro, 32 años).

Aquí claramente se observa que no es por un reclamo del hijo/a que ellos duerman en la cama del padre, sino que el niño/a parecería cubrir un espacio vacante del padre. Este hecho pone de manifiesto las vivencias de estos padres en el proceso posterior al divorcio o separación donde se "desnaturaliza" la imagen del varón "duro" e "insensible".

El dolor por la pérdida, los cambios en la vida cotidiana y las responsabilidades como padre que estos deben asumir en un terreno de cambios, en el cual los roles de género están en transición, invitan a reflexionar sobre la importancia de visibilizar cómo estos padres atraviesan este proceso, más que a seguir naturalizando las construcciones sociales de los géneros desde las cuales se los deja en un lugar de poder absoluto, fortaleza e insensibilidad que oculta sus padecimientos.

\section{Conclusiones}

Es indudable que el siglo XXI trae grandes cambios en lo que respecta a la masculinidad, dado que los cambios en el rol de la mujer han generado movimientos en el posicionamiento de la masculinidad hegemónica. El varón ya no 
es el único sustento económico del hogar, su poder ya no pasa por encarnar un rol activo sexual ni por ser quien hace posible lo más anhelado en la mujer: la maternidad.

Por otro lado, aunque en las últimas décadas la mujer ha ingresado en el mercado laboral, lo que supone una mejora en términos de igualdad, los estereotipos de género en la distribución de las tareas domésticas y de crianza de los hijos aún persisten, por lo que parecería que dichos estereotipos son muy resistentes al cambio.

Al respecto, Beck (1998) afirma que, si bien son notorios los cambios en el rol de la mujer en el ámbito público debido a su incorporación al mercado laboral, los cambios en el ámbito privado se tornan más lentos.

Estos cambios dan cuenta de que las identidades femenina y masculina están en revisión. Los estereotipos tradicionales coexisten en tensión con las transformaciones de estos. Esto ocurre tanto en la construcción subjetiva de la feminidad como de la masculinidad.

El mandato social que identificaba a la mujer como "ama de casa y madre" entra en conflicto con el mandato social actual de "mujer profesional e independiente".

La coexistencia en tensión de estereotipos tradicionales que atribuyen a la identidad masculina la fuerza, el poder y el sustento económico de la familia y nuevas concepciones de masculinidad en proceso de construcción, representa una fuente generadora de conflictos y ambigüedades que se reflejan en la vivencia subjetiva de desconcierto y confusión por parte de los hombres fundamentalmente (Pleck, 1983), aunque esto también involucra a las mujeres, por supuesto.

López de la Vieja (2000) enuncia que estos cambios, los cuales dejan de identificar al hombre con un rol privilegiado caracterizado por la fuerza, el poder, la independencia y la virilidad, son vividos por los hombres como amenazantes, ya que en la medida en que los "viejos" modelos hegemónicos están en vigencia conjuntamente con los nuevos, los enfrentan con el temor a la pérdida de la identidad social internalizada en el proceso de socialización.

El trastrocamiento de valores, creencias, hábitos, roles, posicionamientos y relaciones de poder entre los géneros acarrea alteraciones de la subjetividad, en tanto ha iniciado un proceso de modificación de la imagen de sí y del otro.

Así mismo, los cambios en los roles tradicionales de los varones y mujeres conforman un marco en el cual la situación del divorcio y la modalidad de configurar el vínculo entre padres e hijos e hijas adquiere su matiz particular. 
La función paterna, en lo que respecta a estos padres, está en transición y, por lo tanto, en conflicto y contradicción. Es decir, si bien se mantienen algunos rasgos característicos del estereotipo de masculinidad tradicional, por ejemplo, tendencia a definirse como proveedor, evitar tareas asociadas al estereotipo femenino, mostrarse fuerte, estas conductas coexisten en tensión con la mayor participación en las tareas de crianza, con el hecho de compartir la manutención de los hijos e hijas entre ambos padres y, por último, con la expresión del dolor emocional causado por la pérdida de la cotidianeidad con sus hijos e hijas.

Lo anterior se articula con la dificultad para poner límites a los niños, ya que, al decir de los participantes, los conflictos que esto podría suscitar impiden que disfruten los pocos momentos que comparten, los cuales son altamente valorados por ellos.

Esta reflexión tuvo como finalidad problematizar las construcciones sociales de género que tienen efecto de poder sobre la vida de las personas.

Por un lado, los padres que atraviesan el doloroso proceso postseparación o divorcio invisibilizan sus padecimientos o toman a sus hijos e hijas como fuente de apoyo emocional, ya sea por propios mandatos sobre la masculinidad ideal como por los sociales.

Por otro lado, el clima de cambios y transiciones representa una pérdida de la identidad anterior, lo cual significa una etapa de crisis, contradicciones y conflictos para con los padres mismos, tanto en los que los define como varones como en la paternidad.

\section{Referencias bibliográficas}

ACRICH DE GUTMAN, L. (2003). La ciudad frente al maltrato hacia las personas mayores. En Aizen, R.(Comp.), Temas de Gerontología Social (pp21-36). Buenos Aires: Dirección General de la Tercera Edad, Gobierno de la Ciudad de Buenos Aires.

ARIZA, M. y DE OLIVERA, O. (2001). Familias en transición y marcos conceptuales en redefinición. Papeles de Población, 7(28), 9-39. Recuperado de http://www.redalyc.org/pdf/112/11202802.pdf

BADINTER, E. (1993). XY La identidad masculina. Madrid: Alianza.

BAUMAN, Z. (2008). Amor Líquido: acerca de la fragilidad de los vínculos humanos. Buenos Aires: Fondo de Cultura Económica.

---. (2008). Vida de consumo. Buenos Aires: Fondo de Cultura Económica.

BECK, U. (1998). La sociedad de riesgo. Hacia una nueva modernidad. Barcelona: Paidós.

BERGER, P. y LUCKMANN, T. (1972). La construcción social de la realidad. Buenos Aires: Amorrortu. 
BOWLBY, J. (1989). Una base segura. Buenos Aires: Paidós.

BURIN, M. (2012). Masculinidades y Femineidades: Identidades Laborales en Crisis. En Hazaki, C. (Comp.), La crisis del patriarcado (pp 63-78) Buenos Aires: Topía.

CARRASCO, M. J. y GARCÍA MINA, A. (2005). El ajuste trabajo familia desde una perspectiva de género. Madrid: Universidad Pontificia Comillaz.

CASTELLS, M. (2001). La galaxia internet. Madrid: Cultura Libre

CÓDIGO CIVIL Y COMERCIAL DE LA NACIÓN. Ministerio Justicia y Derechos Humanos, Presidencia de la Nación. Recuperado de http://www.sde.gob.ar/justicia/cuadrocomparativo.pdf

DIRECCIÓN GENERAL DE ESTADÍSTICA Y CENSOS DE LA CIUDAD DE BUENOS AIRES. Recuperado de http://www.estadisticaciudad.gob.ar/eyc/?page_id=1848

FERNÁNDEZ, A. M. (2014). La mujer de la ilusión. Buenos Aires: Paidós.

GARCÍA GARCÍA, A (2009). Modelos de identidad masculina. Recuperado de: Modelos de identidad masculina - EPrints Complutense eprints.ucm.es/9537/ por AA García García - 2009

GIDDENS, A. (1998). Modernidad e identidad del yo. El yo y la sociedad en la época Contemporánea. Barcelona: Península.

GUZMÁN, I. y CONSTENLA, M. (2012). Maternidad y paternidad después del divorcio o separación. Recuperado de http://www.grupoambos.com/articulos/educacion-y-familia/133-parejas-paternidad-y-maternidad-despues-deldivorcio-o-separacion

KIMMEL, M. (2005). Handbook of Studies on Men and Masculinities. London: Sage Publications.

LÓPEZ DE LA VIEJA, M. T. (2000). Feminismo: del pasado al presente. Salamanca: Universidad de Salamanca.

PEDROSA, S. y BOUZA, J. M. (2008). El Síndrome de Alienación Parental. Buenos Aires: García Alonso.

PLECK, J. (1983). El mito de la masculinidad. Cambridge, Massachusetts: The MIT Press.

QUARESMA DA SILVA, D. y ULLOA GUERRA, O. (2013). Estudios de masculinidades en la región oriental de Cuba. Athenea Digital, 13(2), 245-257. Recuperado de http://atheneadigital.net/article/viewFile/v13-n2-quaresmaulloa/1120-pdf-es

SEGARA, M. y CARABÍ, A. (Eds.). (2000). Nuevas masculinidades. Barcelona: Icaria.

WAISBLAT WAINBERG, A. y SÁENZ BERBEJILLO, A. (2001). La construcción sociohistórica de la existencia. Patriarcado, capitalismo $\quad y \quad$ desigualdades instaladas. Recuperado http://www.academia.edu/2558036/LA_CONSTRUCCI\%C3\%93N_SOCIO_HIST\%C3\%93RICA_DE_LA_EXISTENCIA._PATR IARCADO_CAPITALISMO_Y_DESIGUALDADES_INSTALADAS

WATZLAWICK, P., BEAVIN, J., y JACKSON, P. (1986). Teoría de la comunicación humana. Buenos Aires: Tiempo Contemporáneo.

ZICAVO, N. (2006). ¿Para qué sirve ser padre? La padrectomía y el divorcio. Concepción: Ediciones Universidad del BíoBío. 